CHRONIQUE DE LA RECHERCHE

\title{
LE WISSENSCHAFTSKOLLEG DE BERLIN «INSTITUTE FOR ADVANCED STUDY »
}

\begin{abstract}
Ayant eu le privilège de passer un an au Wissenschaftskolleg de Berlin, je ne saurais mieux exprimer ma gratitude envers cette institution exemplaire, et encore mal connue en France, qu'en essayant de la faire un peu mieux connaître de ceux qui, à un titre ou à un autre, pourraient s'y intéresser. Je donnerai d'abord quelques informations de caractère général ; j'y ajouterai ensuite quelques notes d'ordre plus concret et plus personnel.

Le Wissenschaftskolleg, fondé en 1980, a commencé son existence effective en 1981. Le sous-titre anglais qu'il porte officiellement - Institute for Advanced Study - annonce clairement la couleur : la tradition à laquelle il se rattache est celle de son illustre homonyme de Princeton, avec qui il entretient d'ailleurs de nombreux rapports institutionnels et personnels. Il se propose essentiellement de favoriser la recherche scientifique, en invitant chaque année (du début d'octobre à la fin de juillet) une quarantaine de chercheurs de toutes nationalités et de toutes disciplines, et en leur procurant les meilleures conditions de travail pour concevoir, exécuter ou terminer un projet scientifique qu'il appartient à chacun de choisir en toute liberté. Les invitations sont lancées sur la proposition d'un Conseil scientifique, composé de sommités internationales, qui se saisit lui-même des suggestions avancées, soit par les intéressés eux-mêmes, soit le plus souvent par des tiers entretenant quelque relation avec le Wissenschaftskolleg. La plus grande diversité est recherchée dans l'éventail des nationalités: si l'Allemagne et les États-Unis fournissent d'importants contingents, beaucoup d'autres pays d'Europe occidentale et orientale, ou même d'autres continents, sont également représentés. Même diversité dans le choix des professions et des disciplines : si les universitaires d'une part, les sciences humaines de l'autre, fournissent la majorité, leur domination n'est pas exclusive. Les Fellows (telle est leur appellation habituelle) sont également diversifiés par le sexe et par l'âge. Chaque année, cependant, et c'est sans doute là l'un des aspects les plus heureux de sa politique, le Wissenschaftskolleg définit un certain nombre de "centres de gravité » (Schwerpunkte), qui varient d'une année sur l'autre, et autour desquels sont regroupées un petit nombre d'invitations. Chaque Fellow peut ainsi profiter simultané-
\end{abstract}

Revue de synthèse : IVe S. No 4, octobre-décembre 1986. 
ment des contacts qui lui sont offerts avec des collègues dont les intérêts et les champs de recherche sont très différents des siens, et se retrouver en terrain de connaissance à plus petite échelle : aucun ne se sent isolé dans sa propre spécialité, et chaque atome trouve rapidement sa molécule d'accueil, en attendant que la vie en commun fasse se découvrir d'autres affinités et se nouer d'autres regroupements.

Financé pour l'essentiel par la Fondation scientifique Ernst Reuter, ellemême instituée par la Ville et l'État de Berlin et soutenue par diverses institutions scientifiques et fondations privées de Berlin et de la République fédérale, le Wissenschaftskolleg est confortablement installé dans une belle villa de Grunewald, l'un des quartiers les plus agréablement verdoyants de BerlinOuest. Des villas voisines abritent la bibliothèque, le secrétariat et divers services. Les Fellows, rémunérés par le Collège sur une base d'équivalence avec leurs appointements ordinaires, habitent tantôt sur place, s'ils sont venus individuellement, tantôt dans des appartements loués en ville, s'ils sont accompagnés de leur famille. Un nouveau bâtiment, en cours de construction à côté du bâtiment principal, contiendra des bureaux et appartements supplémentaires.

Le Wissenschaftskolleg est soucieux de permettre aux Fellows de profiter au mieux d'une année où ils sont exceptionnellement libérés de leurs tâches d'enseignement, de leurs charges administratives et de leurs responsabilités universitaires; il se garde louablement de substituer de nouvelles obligations à celles dont, grâce à lui, les Fellows se trouvent provisoirement exemptés. Tout ce qui leur est demandé est de donner quelques gages minimaux à la convivialité et aux échanges intellectuels en prenant, dans toute la mesure du possible, leur repas de midi au Collège, et en présentant une fois dans l'année un résumé de leurs travaux devant leurs collègues et (s'ils le souhaitent) quelques invités du milieu intellectuel et culturel de Berlin. Ils peuvent sans difficulté s'absenter quelques jours pour donner des conférences ou participer à des colloques et réunions scientifiques.

Par ailleurs, le maximum est fait sur place, avec des moyens importants et une souriante efficacité, pour faciliter leur travail. Une petite bibliothèque, contenant surtout de grands textes et ouvrages de référence, est gérée par une nombreuse équipe dont les membres peuvent aller, à la demande des Fellows, emprunter des ouvrages plus spécialisés dans l'une ou l'autre des grandes bibliothèques de la ville. Un secrétariat très étoffé, dont le personnel polyglotte est admirablement compétent et doté du matériel le plus moderne, est également à la disposition des Fellows. Des lecteurs de microfilms et des appareils de photocopie perfectionnés sont disposés en abondance dans les couloirs et sur les paliers.

Pour donner maintenant une idée un peu plus concrète du climat qui peut se créer dans une " promotion" du Wissenschaftskolleg, voici quelques indications chiffrées concernant celle à laquelle j'ai eu l'honneur d'appartenir (1984-1985). Sur un ensemble de 38 Fellows (dont 5 femmes), les pays suivants étaient représentés : États-Unis d'Amérique (16 membres), Allemagne de l'Ouest (12), Suisse (3), Pologne (3), Angleterre (1), France (1), Israël (1), Suède (1). Les principaux Schwerpunkte étaient, cette année-là : l'économie politique 
et le droit, la thermodynamique, la slavistique et notamment l'histoire du théâtre russe, l'histoire ancienne et moderne, principalement l'histoire intellectuelle et culturelle, l'histoire de la philosophie ancienne, la musique et la musicologie. La plupart des Fellows étaient des universitaires, mais non pas tous : quelques-uns représentaient les milieux de la création artistique et de la communication culturelle. Au total, comme on le voit, un éventail extrêmement varié, dans lequel les sciences humaines dominaient, sans toutefois écraser les autres, malgré l'attachement plus étroit qui lie la plupart des scientifiques à leurs laboratoires. Les techniques elles-mêmes n'étaient pas absentes du tableau : c'est ainsi que l'un de nos collègues, philosophe passionné d'aérostation théorique et pratique, nous fit en guise de conférence la démonstration d'un moteur de son invention et de sa fabrication, à la fois plus léger et moins bruyant que ses homologues ordinaires. Il faut ajouter que les musiciens du Collège surent, d'autres soirs, éveiller d'autres échos dans la grande salle des conférences, grâce au superbe piano de concert qui fait partie de son mobilier permanent.

Entre bruit et musique, disons un mot de la langue, sujet possible de préoccupation pour les Fellows à venir, et sujet réel d'anxiété pour plus d'un Fellow passé. L'allemand est, comme il est naturel, la langue officielle du Wissenschaftskolleg ; les documents internes et les annonces publiques s'y font exclusivement dans cette langue, et il est recommandé d'en avoir quelque teinture au moins; la vie quotidienne n'étant pas totalement prise en charge par la communauté du Collège, il faut aussi pouvoir se débrouiller tant bien que mal pour en résoudre les petits problèmes au jour le jour. L'anglais sert cependant de véhicule de secours à beaucoup d'échanges. La plupart des intellectuels allemands le parlent à merveille ; la connaissance du français est moins répandue, mais elle étonne souvent par sa perfection chez ceux qui la possèdent. Il faut cependant compter avec la coquetterie d'un bon nombre de Fellows non allemands, qui mettent leur point d'honneur à s'exprimer, plus d'une fois avec une grande aisance, dans la langue de Goethe. Au total, on pourrait recommander au futur Fellow de pratiquer à la fois l'allemand et l'anglais, et très bien l'une au moins de ces deux langues. Si c'est l'anglais, la vie à Berlin lui permettra de réduire le déséquilibre, et ses éventuels progrès seront sympathiquement appréciés.

La description de cette Abbaye de Thélème en plein Berlin ne serait pas exacte, en effet, si l'on omettait de mentionner qu'elle ne vit pas en vase clos. Grâce aux liens qu'entretient le Wissenschaftskolleg avec les institutions universitaires, scientifiques, culturelles de Berlin, les Fellows ont de multiples occasions de nouer des liens en dehors du Collège avec beaucoup des personnalités intéressantes dont la ville regorge; et la carte de visite du Collège sert de bonne introduction dans une population dont, au reste, l'hospitalité et l'ouverture sont remarquables. Sur un autre plan, les Fellows ont le rare privilège de pouvoir se procurer, au Collège même et par son intermédiaire, des billets d'entrée dans tous les théâtres, opéras, salles de concert de la ville; et tous ceux qui ont essayé de pénétrer dans la salle de la Philharmonie par les voies ordinaires savent ce que représente ce privilège. Celui même, s’il en était, qui 
ne mettrait pas les pieds en dehors du Collège ne manquerait pas d'y vivre divers événements grands ou petits, entre le bal offert aux Fellows par le personnel du Collège, au début de l'année, et celui offert au personnel du Collège par les Fellows à la fin de l'année ; les murs y servent à l'accrochage de plusieurs expositions temporaires; et il ne se passe guère de jour sans que l'on annonce la présence, à la table commune, de quelque hôte de marque. Enfin, les groupes de Fellows qui travaillent dans le même domaine ont la possibilité d'organiser sur place, avec le concours administratif et financier du Collège, des colloques auxquels ils peuvent convier un nombre appréciable de leurs collègues extérieurs. À quoi s'ajoute la ronde des parents, des enfants, des amis, qui tisse autour de chaque Fellow un réseau d'allées et venues entre le Collège et le vaste monde qui l'entoure.

Disons pour finir qu'en dépit de l'euphorie qui envahit assez rapidement la plupart d'entre eux lorsqu'ils prennent contact avec leur nouveau cadre de vie, les membres du Wissenschaftskolleg n'y subissent pas une métamorphose totale : fidèles à leur nature et à leurs habitudes antérieures, ils travaillent tous beaucoup, et beaucoup travaillent énormément. Mesurée en livres, articles, communications de toute sorte, la productivité globale du Collège est assez impressionnante, même sì tous les fruits n'en viennent pas simultanément à maturité. On peut en avoir un avant-goût en consultant le Jahrbuch dans lequel chaque année, les Fellows réunissent des échantillons ou des résumés de leurs travaux. Ce qu'il faut dire aussi, c'est que le Collège ne leur permet pas seulement de donner à leur recherche personnelle un coup d'accélérateur souvent décisif ; il favorise aussi la genèse de travaux qui sans lui n'auraient sans doute jamais vu le jour, notamment des travaux collectifs et interdisciplinaires. En dépit des critiques que peut toujours s'attirer une institution qui ne se cache pas d'être élitaire, sinon élitiste, de tels résultats sont de ceux dont peuvent s'enorgueillir le recteur Wapnewski, directeur du Collège depuis sa fondation, et l'admirable équipe administrative qu'il a su réunir. Dans cette ville dont le passé est effroyable et dont le présent est tragique, à quelques pas du Mur, et du bunker de Hitler, c'est l'Allemagne des Humboldt qui revit véritablement. Le climat intellectuel et humain que le Wissenschaftskolleg a réussi à créer en quelques années est tel que chacun de ceux qui en ont bénéficié, loin de savourer sa chance dans le silence et la mauvaise conscience, ne rêve que d'en favoriser l'extension et d'en multiplier l'écho.

Pour cette raison, et aussi parce que je sais que le Wissenschaftskolleg désire particulièrement développer ses relations avec la France, je me borne, en guise de conclusion, à donner son adresse : Wallotstrasse 19, D-1000 Berlin 33 .

Jacques BRUNSCHWIG, Université de Paris $X$-Nanterre. 